\author{
Manuscript published as: \\ Scripta Materialia, Volume 132, 15 April 2017, Pages 13-16 \\ http://dx.doi.org/10.1016/j.scriptamat.2017.01.020 \\ (copyright Elsevier Science)
}

\title{
Occurrence of the Portevin Le-Châtelier effect in open-cell microcellular Al-2 wt\% Mg
}

\author{
M. Knapek ${ }^{\mathrm{a}^{*}}$, P. Dobroňa, K. Máthis ${ }^{\mathrm{a}}$, K. Illkováb1, A. Mortensen ${ }^{\mathrm{b}}$, \\ F. Chmelík
}

aDepartment of Physics of Materials, Faculty of Mathematics and Physics, Charles University, Ke Karlovu 5, 12116 Prague 2, Czech Republic

bLaboratory of Mechanical Metallurgy, École Polytechnique Fédérale de Lausanne, Station 12, CH1015 Lausanne, Switzerland

*corresponding author: knapek@karlov.mff.cuni.cz

\section{Abstract}

Microcellular open-cell metal foams with pores of 75 or $400 \mu \mathrm{m}$ in diameter and made of pure $\mathrm{Al}$ or $\mathrm{Al}-2 \mathrm{wt} \% \mathrm{Mg}$ are tested in compression. The aluminum foam exhibits a typical smooth plastic flow and a positive strain rate sensitivity, whereas plastic instabilities and a negative strain rate sensitivity appear in the stress-strain curves of the Al- $2 \mathrm{wt} \% \mathrm{Mg}$ foams, indicating the presence of the Portevin Le-Châtelier (PLC) effect. The effect is further confirmed by acoustic emission analysis. Taken together, the data indicate that the PLC effect is caused in this material by the sudden collective deformation of many struts across the material.

\footnotetext{
${ }^{1}$ Present address: Department of Materials Engineering, Institute of Plasma Physics, Czech Academy of Sciences, 18200 Prague 8, Czech Republic
} 
Keywords: Portevin Le-Châtelier effect; metal foams; strain rate sensitivity; acoustic methods; plastic deformation.

Artificial cellular materials have been made of metal, and are then often simply called "metal foams". Cellular metals are attractive in lightweight structures, when specific features of porous materials combine advantageously with their structural properties to produce materials for applications in, e.g., mechanical damping, vibration control, energy absorption, heat exchange, or filtering [1-3]. Most microcellular metallic materials available on the market have closed cells, as this is advantageous for structural performance; these are often true "metal foams", in the sense that their pores are former bubbles, which were produced by foaming a metal slurry. As a result of the stochastic process by which the pores were produced, their structure generally features inhomogeneities in the density, in the shape and in the size of the cells they contain. Commercial open-pore cellular metals, on the other hand, can be made to feature monosized and relatively regular pores. The mechanical response of these materials is, in turn, more predictable and reproducible [1,4].

The salt-replication technique [1] is one of the processes by which open-pore microcellular metals can be produced, with tight control of the pore size, volume fraction and shape, and also of the nature of the metal of which the "foam" is made. This process was utilized for the production of open-pore metal foams investigated in this work; our main goal here is to assess the difference in compressive deformation behavior between microcellular aluminum and microcellular aluminum containing $2 \mathrm{wt} \%$ of magnesium (Al2Mg). Interest in this comparison comes from the fact that most bulk aluminum-magnesium alloys are known to exhibit the Portevin-Le Châtelier (PLC) effect - a plastic instability appearing as serrations on the deformation curves which, in general, negatively affects the mechanical properties. To our knowledge, the PLC effect in metal foams has so far not been studied. 
Test samples of open-cell microcellular aluminum (Al, $99.99 \%$ purity) and Al2Mg were produced using salt-replication which, in brief, comprises: (i) producing a preform of coldpressed $\mathrm{NaCl}$ particles, (ii) infiltrating this preform with pressurized metal, (iii) solidifying the metal within the preform, (iv) machining the resulting preform/metal composite to give it its final shape, (v) leaching the preform in water. Generally, monosized $\mathrm{NaCl}$ particles are used, and leaching is conducted in the presence of a corrosion inhibitor; for more details see [5]. Main characteristics of samples investigated in this work are listed in Tab. 1. As a result of many factors (particle size, infiltration pressure, etc.) affecting the volume fraction of solid $V_{\mathrm{s}}$, this quantity varies between different sets of samples. On the other hand, there is only a small variation in all structural parameters, including $V_{s}$, within individual sample sets, as all compression samples of a set were machined from the same infiltrated ingot.

Tab. 1. Main characteristics of Al and Al2Mg foam samples investigated in this work.

\begin{tabular}{|l|l|l|l|}
\hline Material & Pore size $[\boldsymbol{\mu m}]$ & Diameter $\times$ height $[\mathbf{m m}]$ & $\boldsymbol{V}_{\mathbf{s}}[\mathbf{\%}]$ \\
\hline \multirow{2}{*}{$\mathrm{Al}$} & 400 & $20 \times 20$ & $24.5-25.9$ \\
\cline { 2 - 4 } & 75 & $10 \times 20$ & $29.9-31.7$ \\
\hline \multirow{2}{*}{$\mathrm{Al} 2 \mathrm{Mg}$} & 400 & $10 \times 20$ & $22.8-23.7$ \\
\cline { 2 - 4 } & 75 & $10 \times 20$ & $30.8-33.1$ \\
\hline
\end{tabular}

Compression tests were performed using a universal testing machine (Instron 5882) at room temperature with a crosshead speed of $10^{-3} \cdot \mathrm{s}^{-1}$. The acoustic emission (AE) response was monitored with a Physical Acoustics Corporation (PAC) PCI-2 board and a broadband AE sensor. The preamplifier (PAC) gain was set to $60 \mathrm{~dB}$. The AE streaming data were recorded with a sampling rate of $2 \mathrm{MHz}$.

The compressive stress-strain curves of each set (Fig. 1) almost superimpose, showing a good reproducibility of the material behavior. This can be directly related to the fact mentioned above, namely that all parameters (including the volume fraction of solid, $V_{\mathrm{s}}$ ) are 

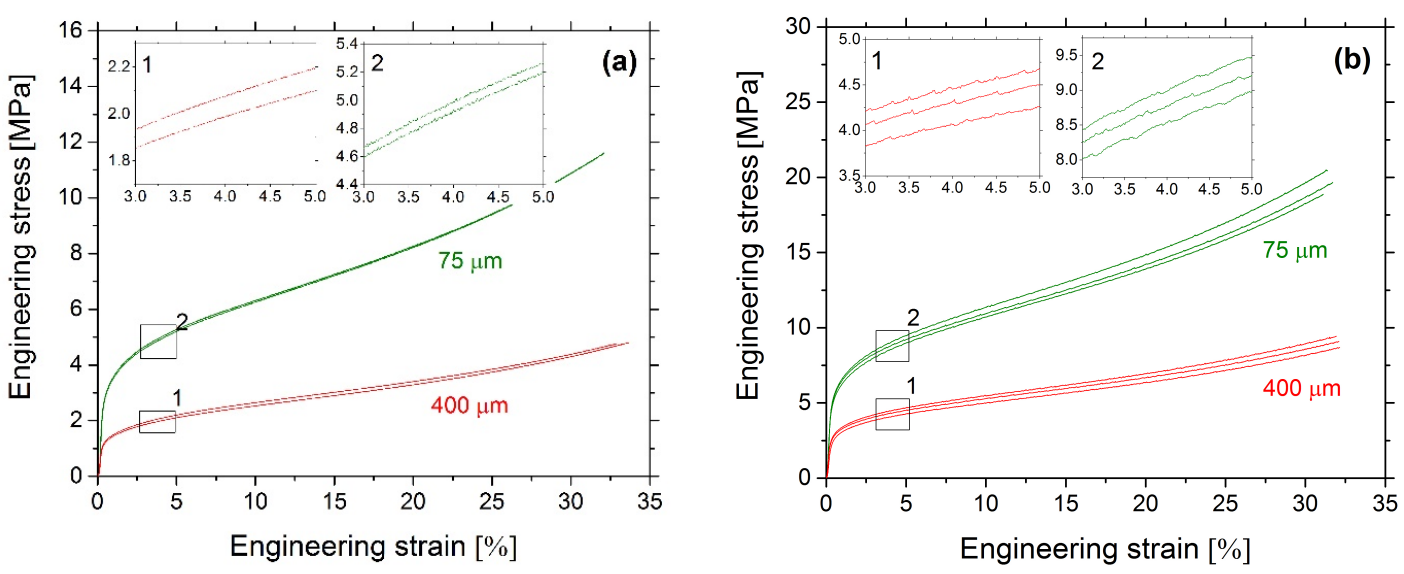

Fig. 1. Compressive curves of (a) Al foam samples (b) Al2Mg foam samples, both with pore sizes of $400 \mu \mathrm{m}$ and 75 um. Samples Al2 Mg exhibit a serrated plastic flow, suggesting the presence of the PLC effect.

rather constant across samples of each set. The curves of the pure Al foam samples (Fig. 1a) are smooth, as is characteristic of replicated microcellular foams (but unlike most commercial closed-cell metal foams). Turning to the Al2Mg microcellular foams, one finds broadly similar stress-strain curves, with the difference that a close-up on the stress-strain curves reveals the presence of many serrations, Fig. $1 \mathrm{~b}$, similar to serrations that were observed in earlier work on replicated Al5Mg foams [6]. Such saw-tooth curves are a typical signature of the PLC effect. Two features characterize the PLC effect. The first is a negative strain rate flow stress sensitivity $S$, Eq. 1 .

$$
S=\frac{\partial \sigma}{\partial \dot{\varepsilon}}<0
$$

where $\sigma$ is the flow stress and $\dot{\varepsilon}$ is the strain rate. The presence of a negative strain rate sensitivity is often ascertained by repeating the deformation experiments on additional samples at different strain rates. In the case of microcellular metals, however, this might be inconclusive as their structures and hence their flow stress are somewhat stochastic in nature. For this reason, strain-rate jump tests were carried out on the microcellular metal samples; results are shown in Fig. 2. 

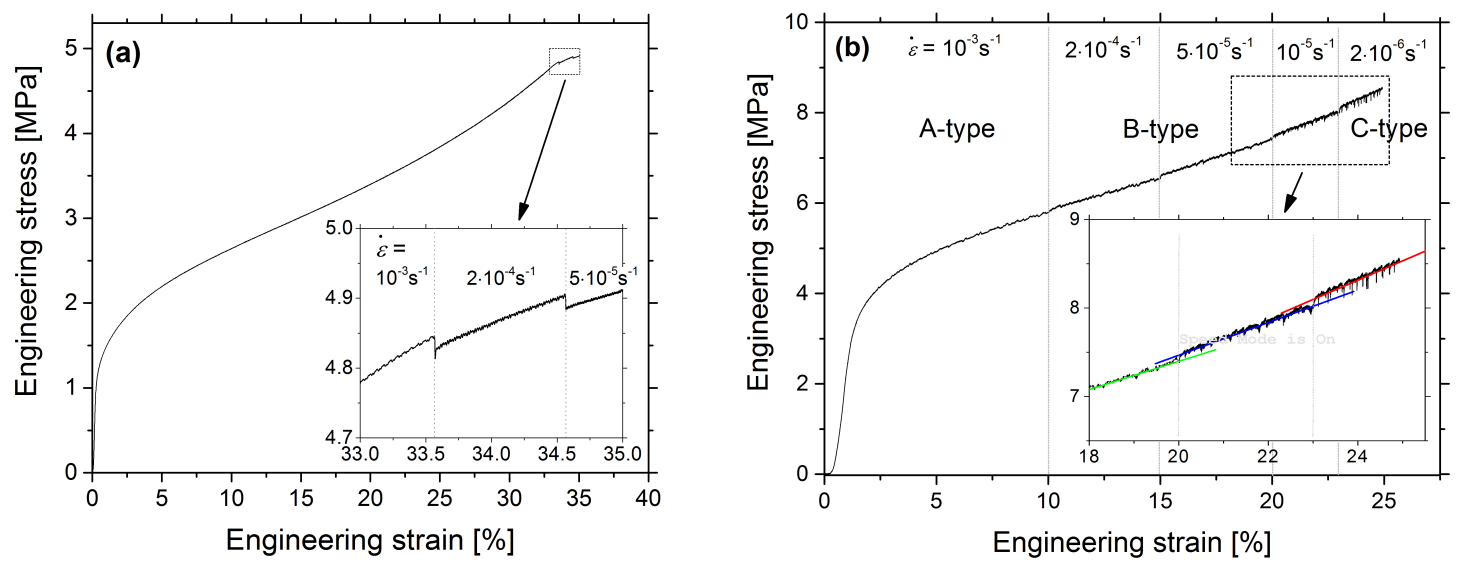

Fig. 2. (a) "Normal" (positive) strain-rate sensitivity observed in pure Al $400 \mu \mathrm{m}$ foam sample, (b) Negative strain-rate sensitivity manifesting PLC effect in Al2 Mg 400 um foam sample.

In Fig. 2a, which shows results from a strain-rate jump test conducted on microcellular $\mathrm{Al}$ with $400 \mu \mathrm{m}$ diameter pores, one observes a conventional, positive strain-rate dependence of the flow stress, which decreases each time the displacement rate is decreased. Figure $2 \mathrm{~b}$ shows corresponding results for microcellular $\mathrm{Al} 2 \mathrm{Mg}$, again with pores $400 \mu \mathrm{m}$ in diameter: the initial strain rate of $10^{-3 \cdot} \cdot \mathrm{s}^{-1}$ was gradually decreased in four steps to a final strain rate as low as $2 \cdot 10^{-6} \cdot \mathbf{s}^{-1}$. The negative strain rate sensitivity is evidenced by the fact that the flow stress rises each time the compression rate is decreased. Moreover, a transition from A-type PLC to B- and finally C-type is evidenced, which is another clear manifestation of the PLC effect.

A second signature of the PLC effect can be detected using Acoustic Emission (AE) tests. In the present mechanical tests, the AE signal was recorded during the deformation and analyzed to detect whether it contains typical features of the AE signal recorded in bulk metals exhibiting the PLC effect. The AE signal median frequency was evaluated as it has been shown to be a credible indicator of correlations in collective dislocation dynamics $[7,8]$. The time-window used for the calculation of median frequency was $500 \mu$ s; the data were smoothed using Savitzky-Golay filter. The evolution of the AE median frequency during the test on the microcellular Al2Mg $400 \mu \mathrm{m}$ sample is shown in Fig. 3 (all three tested 

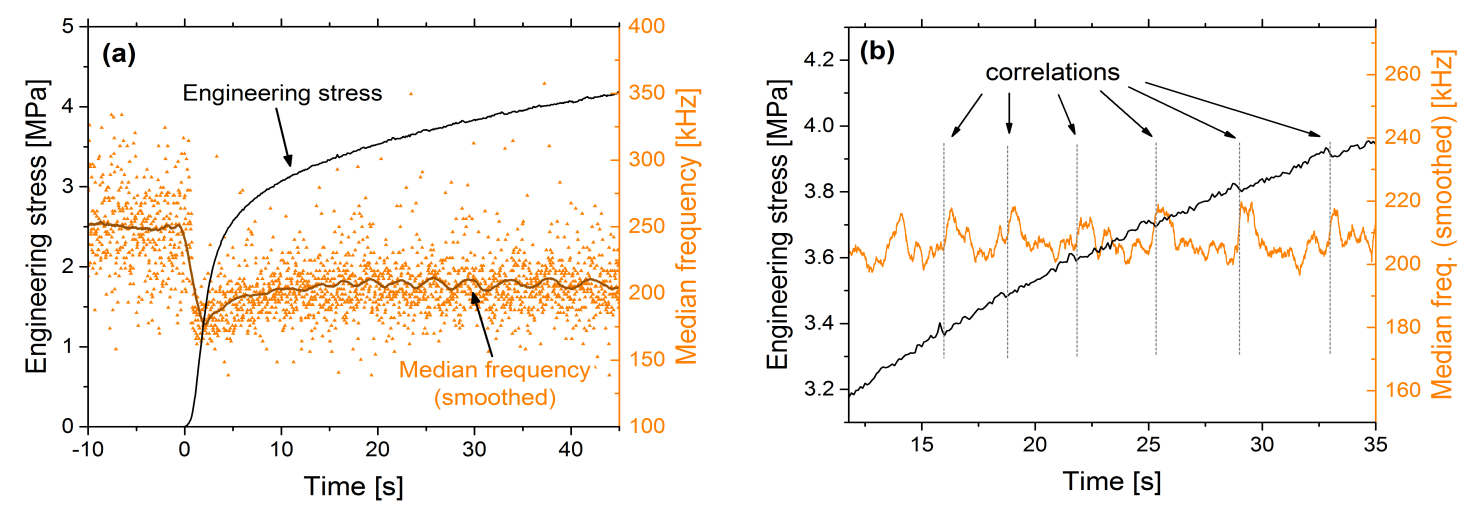

Fig. 3. Median AE frequency evolution in the early stage of compression of Al2Mg $400 \mu \mathrm{m}$ foam sample.

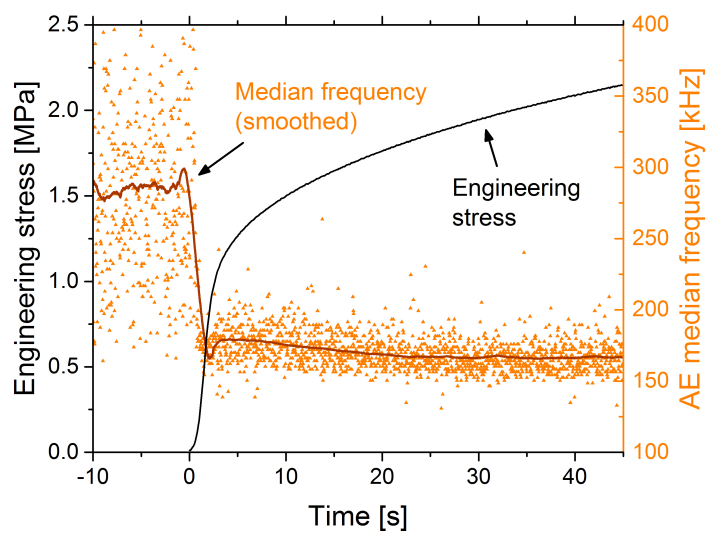

Fig. 4. Median AE frequency evolution in the early stage of compression of pure Al $400 \mu \mathrm{m}$ foam sample.

samples exhibited very similar characteristics). The AE signal was recorded starting several seconds before the actual test in order to gather information about the noise background. At the beginning of compression, the AE median frequency decreases, starting from a relatively high magnitude in the wide-spectrum noise region. It can be seen that the median frequency exhibits fluctuations during compression of the sample. As shown in Fig. 3b, these oscillations are directly and quite precisely correlated to the PLC serrations on the deformation curve. The reduction in median frequency is typically attributed to enhanced correlations in dislocation processes and strain localization [7-9], and this observation agrees with similar investigations on bulk Al5Mg samples [7,9]. On conducting similar experiments with microcellular samples of pure aluminum, no such fluctuation were observed, Fig. 4. We thus conclude that the PLC effect is observed when an alloy (Al2Mg) 
known to exhibit the effect in bulk form takes the form of a microcellular, open-cell replicated foam.

The PLC effect is a plastic instability observed in dilute alloys, caused by a pinningdepinning interaction between dislocations and solute atoms. It is one of the few cases where the heterogeneous nature of plastic flow shows up at the macroscopic scale. In bulk samples, the effect consists of repetitive localization of plastic strain within transversal deformation bands that traverse the entire specimen $[7,10]$. Therefore, it is at first glance intriguing that the PLC effect can also occur in a complex, highly porous, microcellular material made of many struts that each deform, a priori, separately.

An intermittency in the plastic flow, which is a result of slip events caused by a collective movement of large dislocation ensembles (so-called dislocation „avalanches“), has been documented by the $\mathrm{AE}$ technique to exist even if the deformation curves are macroscopically smooth (observed e.g. in metal single-crystals [11], or crumpled aluminum foils [12] whose structural features are, in addition, similar to those of metal foams). In bulk polycrystals, the magnitude of such slip events is limited to one grain (typically tens of micrometers) [13]. Therefore, the scale of such slip events is similar to those observed in the $\mathrm{Al}$ foam or between stress serrations in the Al2 Mg foam.

It is, however, unlikely that the serrations and $\mathrm{AE}$ signal recorded in this study in $\mathrm{Al} 2 \mathrm{Mg}$ foam be caused by the deformation consisting of slip events within individual struts, because: (i) the serrations in the curves are observed macroscopically and their intensity is the same for the 400 and the $75 \mu \mathrm{m}$ diameter foams (Fig. 1b; if serrations were caused by isolated struts they would be roughly 5 times smaller in the $75 \mu \mathrm{m}$ pore size foam); (ii) the collapse of a single strut would not produce an AE signal modulations of the magnitude recorded here (Fig. 3). These observations thus suggest that the PLC effect recorded with the microcellular $\mathrm{Al} 2 \mathrm{Mg}$ material is caused by the collective unstable deformation of many 
struts. This in turn suggests that the material can be seen as a system in a critical state $[7,14]$, in which the stress relaxation from piled-up pinned dislocations within one individual strut can (as a result of the negative strain-rate sensitivity of the material) propagate by causing an abrupt change in local stresses in the neighboring struts, inducing in this way a "chain reaction" generation of nearly simultaneous strain bursts within a considerable number of struts across the sample. The PLC effect in microcellular materials is, therefore, likely to have features in common with several other critical phenomena in materials science, such as the damage-induced fracture of composite materials, which should make it an interesting subject for further enquiry.

To conclude, when deformed in compression, Al2Mg replicated open-cell microcellular foam with a mean pore size of 400 or $75 \mu \mathrm{m}$ displays clear manifestations of the PortevinLe Châtelier effect, namely (i) serrations in the flow curve, (ii) a negative strain rate sensitivity in its plastic flow stress and (iii) an AE signal with frequency fluctuations concomitant to the serrations on the stress signal. These effects are absent in similarly processed and tested replicated microcellular foam of pure aluminum. Analysis of the data leads to conclude that this PLC effect results from the "chain-like" collective deformation of many individual struts across the material.

This work was financially supported by the Czech Science Foundation (grant No. 1510821S), and the award of a Sciex-NMS ${ }^{\mathrm{CH}}$ postdoctoral exchange grant to one of the authors (KI; project code number 13.239).

\section{REFERENCES}

[1] J.F. Despois, PhD thesis, 2005, École polytechnique fédérale de Lausanne, Switzerland. 
[2] A. Kennedy, Porous metals and metal foams made from powders, in: K. Kondoh, (Ed.), Powder Metallurgy, InTech, Nottingham, 2012, pp. 31.

[3] M.F. Ashby, A.G. Evans, N.A. Fleck, L.J. Gibson, J.W. Hutchinson, H.N.G. Wadley, Metal foams: a design guide, Materials \& Design, 2000.

[4] R. Goodall, A. Mortensen, Porous metals, in: D. Laughlin and K. Hono (Eds.), Physical Metallurgy, fifth ed., Elsevier, Amsterdam, 2014, pp. 2399.

[5] Y. Conde, J.S. Despois, R. Goodall, A. Marmottant, L. Salvo, C. San Marchi, A. Mortensen, Adv. Eng. Mater. 8 (2006) 795.

[6] F. Diologent, R. Goodall, A. Mortensen, Acta Mater. 59 (2011) 6869.

[7] I. Shashkov, PhD thesis, 2012, Université de Lorraine, Metz, France.

[8] A. Vinogradov, A. Lazarev, Scripta Mater. 66 (2012) 745.

[9] I.V. Shashkov, M.A. Lebyodkin, T.A. Lebedkina, Acta Mater. 60 (2012) 6842.

[10] A. Yilmaz, Sci. Technol. Adv. Mater. 12 (2011) 063001.

[11] J. Weiss, T. Richeton, F. Louchet, F. Chmelík, P. Dobroň, D. Entemeyer, M. Lebyodkin, T. Lebedkina, C. Fressengeas, R.J. McDonald, Phys. Rev. B 76 (2007) 224110.

[12] M. Abobaker, O. Bouaziz, M. Lebyodkin, T. Lebedkina, I.V. Shashkov, Scripta Mater. 99 (2015) 17.

[13] T. Richeton, J. Weiss, F. Louchet, Acta Mater. 53 (2005).

[14] P. Bak, C. Tang, K. Wiesenfeld, Phys. Rev. A 38 (1988) 364. 